\title{
Efecto de parámetros de mínima cantidad de lubricación en reducción de desgaste de herramienta
}

\section{Effect of Minimum Quantity Lubrication parameters on tool wear reduction}

\author{
MARTÍNEZ-RAMÍREZ, Israel†*, GUTIÉRREZ-RIVERA, Miguel Ernesto, ESPINOZA-TORRES, Isaí \\ y RODRÍGUEZ-FLORES, Javier
}

Universidad de Guanajuato, División de Ingenierías Campus Irapuato-Salamanca.

ID 1 ${ }^{\text {er }}$ Autor: Israel, Martínez-Ramírez / ORC ID: 0000-0002-8186-4390, CVU CONACYT ID: 334143

ID $1^{\text {er }}$ Coautor: Miguel Ernesto, Gutiérrez-Rivera / ORC ID: 0000-0002-6210-4484, CVU CONACYT ID: 3301069

ID $2^{\text {do }}$ Coautor: Isaí, Espinoza-Torres / ORC ID: 0000-0002-9933-5569, CVU CONACYT ID: 907269

ID $3^{\text {er }}$ Coautor: Javier, Rodríguez-Flores / ORC ID: 0000-0001-8486-4735

DOI: $10.35429 / J M E .2019 .10 .3 .19 .26$

Recibido 20 Abril, 2019; Aceptado 30 Junio, 2019

\section{Resumen}

El objetivo del presente trabajo es determinar los parámetros que afectan la efectividad del proceso de mínima cantidad de lubricación (MQL) en el desgaste de flanco durante fresado frontal. Además de determinar los factores, se determinarán los valores en los cuales el MQL es más efectivo para disminuir el desgaste en fresado frontal a velocidad de corte relativamente alta. Mediante un diseño de experimentos de dos niveles y tres factores con dos repeticiones, se efectuaron pruebas de desgaste en insertos grado P45 durante el fresado frontal a alta velocidad de corte ( $900 \mathrm{~m} / \mathrm{min})$ de AISI 1045. Se utilizó un sistema comercial MQL con lubricante no soluble en agua de base vegetal. Los factores analizados fueron el ángulo vertical, cantidad de lubricante y flujo de aire. Los resultados muestran que la cantidad de lubricante y especialmente el ángulo vertical, son factores que afectan en gran medida la efectividad el proceso. Se obtuvo una ecuación mediante la cual se generó la superficie de respuesta para visualizar las zonas en las que los factores dan como resultado un menor desgaste. Se observó 7 veces menos desgaste de flanco al utilizar nivel bajo de flujo de aire, bajo ángulo vertical y un alto flujo de lubricante.

\begin{abstract}
The objective of this work is to determine the influence of Minimum Quantity Lubrication (MQL) parameters on flank wear during face milling. Furthermore, the values of each factor in which the MQL resulted to be effective were determined. A designed experiment with two level, three factor and two replicas was used to test tool wear on $\mathrm{P} 45$ grade inserts at relatively high cutting speed ( $900 \mathrm{~m} / \mathrm{min})$ on steel AISI 1045. A commercial MQL system was used with vegetable base lubricant non soluble in water. Results show that amount of lubricant and vertical angle are key factors that affects the effectiveness of the process. A response surface equation was obtained in order to determine the zones in which the factors resulted in the lowest tool wear. A flank wear decrease of 7 times, respect to the maximum observed, was found by using low level of air flow, low level of vertical angle and high level of lubricant.
\end{abstract}

\footnotetext{
* Correspondencia al Autor (Correo electrónico: israel.martinez@ugto.mx)

$\dagger$ Investigador contribuyendo como primer Autor.
} 


\section{Introducción}

Durante el proceso de maquinado, se produce calor como consecuencia de la fricción entre el material de trabajo y la herramienta de corte y por la deformación plástica que sufre el material en bruto. Dicho calor trae como consecuencia que mecanismos de desgate tales como desgaste químico o por adhesión sean activados (Childs, Maekawa, Obikawa, \& Yamane, 2000). El desgaste por abrasión, aunque no es directamente asociado por la temperatura, al disminuir la dureza de la herramienta con el calor, también tiende a aumentar. El método más comúnmente usado para lidiar con la fricción y el calor es el llamado "por inundación".

En este método chorros de fluido de corte son aplicados por una o más boquillas durante el proceso de corte. En este proceso mucho del fluido de corte se desperdicia al no entrar en contacto directo con la zona de contacto entre la herramienta y el material. Un nuevo proceso surgió en los 90's primeramente desarrollado en Alemania y Japón ( (Heisel, Lutz, Spath, Wassmer, \& Walter, 1994)- (Wakabayashi, Sato, \& Inasaki, 1998)) con la finalidad de conseguir el efecto lubricante pero a un menor costo ambiental y de salud para el operario. Dicho proceso es conocido como mínima cantidad de lubricación (MQL, por sus siglas en inglés). En dicho proceso, bajas cantidades de lubricante son aplicadas por pulverización. Este proceso es considerado como una tecnología verde o limpia que consume menos recursos y es más amigable con el medio ambiente.

Desde que se expandió el conocimiento sobre MQL, ha sido y continúa siendo objeto de investigación. Principalmente en el efecto que tiene sobre el desgaste de la herramienta y, por tanto, en el acabado superficial. Coz et al. usaron MQL interno en taladrado de aleaciones para la industria aeroespacial (Le Coz, Marinescu, Devillez, Dudzinski , \& Velnom, 2012). Bruni et al. modelaron la rugosidad superficial en fresado frontal usando MQL (Bruni, d Apolito, Focellese, Gabrielli, \& Simoncini, 2008). Kasim et al. usaron diseño de experimentos (metodología de superficie de respuesta) para predecir la rugosidad superficial en fresado bajo MQL (Kasim, Che Haron, Ghani, \& Sulaiman, 2013). Mia et al. estudiaron el impacto que tiene la tasa de flujo de MQL en la rugosidad de acero endurecido después de fresado (Mia, Al Bashir, Kan, \& Dhar, 2017).
En el 2016, Sharma et al. publicaron un artículo de revisión con trabajos relacionados con el efecto de diferentes fluidos de corte, convencionales y nanofluidos aplicados bajo condiciones de MQL en el proceso de corte (Sharma, Tiwari, \& Dixit, 2016).

Jang et al. optimizaron parámetros de corte para minimizar la energía de corte en fresado usando MQL (Jang, Jung, \& Seok, 2016). Singh et al. llevaron a cabo un trabajo similar referente a la optimización de parámetros de corte en fresado de Inconel 718, minimizando el desgaste de flanco (Singh, Gupta, Mia, \& Sharma, 2018). Cabe mencionar que parámetros de corte se refiere a profundidades, avances y velocidades de corte, más no se refiere en sí a los parámetros propios del sistema MQL. Relativo a parámetros MQL, Wang et al. investigaron el efecto de la posición de la boquilla en el plano horizontal en el fresado frontal de Inconel 182 (Wang, Cheng, An, Wang, \& Zhu, 2014). Contra la lógica, obtuvieron mejores resultados en la posición cuando el cortador sale del material de trabajo en lugar de cuando entra a cortar. Tawakoli et al. investigaron el efecto de parámetros de MQL aplicado a rectificado (Tawaloki, Hadad, \& Sadeghi, 2010).

Liu et al. publicaron resultados del efecto de diferentes parámetros de MQL en el fresado de Ti-6Al-4V (Liu, Cai, Chen, \& An, 2011). En su trabajo analizaron el efecto que tiene la presión del aire, cantidad de lubricante, y posición horizontal de la boquilla en fuerzas y temperaturas de corte. En el análisis que realizaron, no siguieron una metodología sistemática como lo es el diseño de experimentos, sino que el análisis se basó en evaluar los efectos principales con varios niveles de los diferentes factores (no el mismo número de niveles en cada factor).

Uno de los contras del proceso MQL es la susceptibilidad del proceso a factores tales como la cantidad de lubricante, flujo de aire y posición de la boquilla respecto al movimiento de la herramienta. Para que el proceso tenga el efecto deseado, dichos parámetros deben de ser estudiados. Los trabajos publicados hasta ahora relacionados con el tema del efecto de los parámetros de MQL son pocos comparados con los disponibles sobre el efecto general de MQL sobre rugosidad, desgaste, fuerzas y temperatura de corte. 
En el presente trabajo se propone un diseño de experimentos que consiste en variar los parámetros previamente mencionados. Se diseñó un dispositivo para lograr el ángulo vertical y horizontal lo más preciso posible, el cual es controlado por motores a pasos.

En la parte de métodos y materiales, se describe la metodología de experimentación, condiciones de corte, materiales de la pieza de trabajo y la herramienta.

En la parte de resultados y discusión se presenta el análisis de varianza del diseño de experimentos, fotografías tomadas del desgaste y del montaje de los dispositivos utilizados durante la experimentación. Además, se presenta una explicación de los resultados y su implicación en la aplicación práctica y utilidad para otros investigadores.

\section{Métodos y materiales}

Placas de acero al carbón AISI 1045 fueron preparadas en dimensiones aproximadas a 100 $\mathrm{mm}$ de ancho, $109 \mathrm{~mm}$ de largo y $50.8 \mathrm{~mm}$ de alto para lograr una longitud de corte constante en cada prueba. La longitud de corte se calcula fácilmente de acuerdo del análisis de la Figura 2. El análisis se puede dividir en dos partes: La primera parte cuando la herramienta entra y llega hasta una distancia igual a $L-a$ y la segunda cuando la herramienta avanza de $L-a$ a $L+a$. está dada por:

Para la primera parte la longitud de corte

$s_{1}=\sum_{i=1}^{n_{1}} 2 \cdot r\left[\cos ^{-1}\left(1-\frac{i \cdot f}{r}\right)\right]$

Si $n_{l}$ representa el número de cortes hasta llegar a $L-a$, entonces $n_{l}$ está dada por:

$n_{1}=\frac{L-a}{f}$

en donde $f$ es el avance en $\mathrm{mm} /$ diente y $r$ es el radio del planeador en $\mathrm{mm}$.

Para la segunda parte se tiene que:

$s_{2}=\sum_{j=1}^{n_{2}}\left\{2 \cdot r\left[\frac{1}{2} \cos ^{-1}\left(1-\frac{1}{2}\left(\frac{b}{r}\right)^{2}\right)-\right.\right.$

$\left.\left.\cos ^{-1}\left(1-\frac{j \cdot f}{r}\right)\right]\right\}$ donde $b$ es el ancho de la pieza de trabajo y $n_{2}$ está dada por:

$n_{2}=\frac{a}{f}$

La longitud de corte total por pasada es, por lo tanto, la suma de $s_{1}$ y $s_{2}$.

El radio del planeador es igual a $127 \mathrm{~mm}$, $f$ representa el paso igual a $0.1 \mathrm{~mm}$. Sustituyendo, la longitud de corte por pasada resultó ser $137 \mathrm{~m}$ y el número de veces que la herramienta entra y sale $\left(n_{1}+n_{2}\right)$ es 1092 . Una serie de pruebas iniciales se llevaron a cabo con el fin de evaluar el desgaste a las velocidades recomendadas por el fabricante de herramienta $(250 \mathrm{~m} / \mathrm{min})$. Sin embargo, se observó que el desgaste era poco visible o nulo inclusive a longitudes de corte superiores a los $5 \mathrm{~km}$. Por lo que de acuerdo a la disponibilidad de material se eligió usar una velocidad de corte, $V_{c}$ igual a 900 $\mathrm{m} / \mathrm{min}$

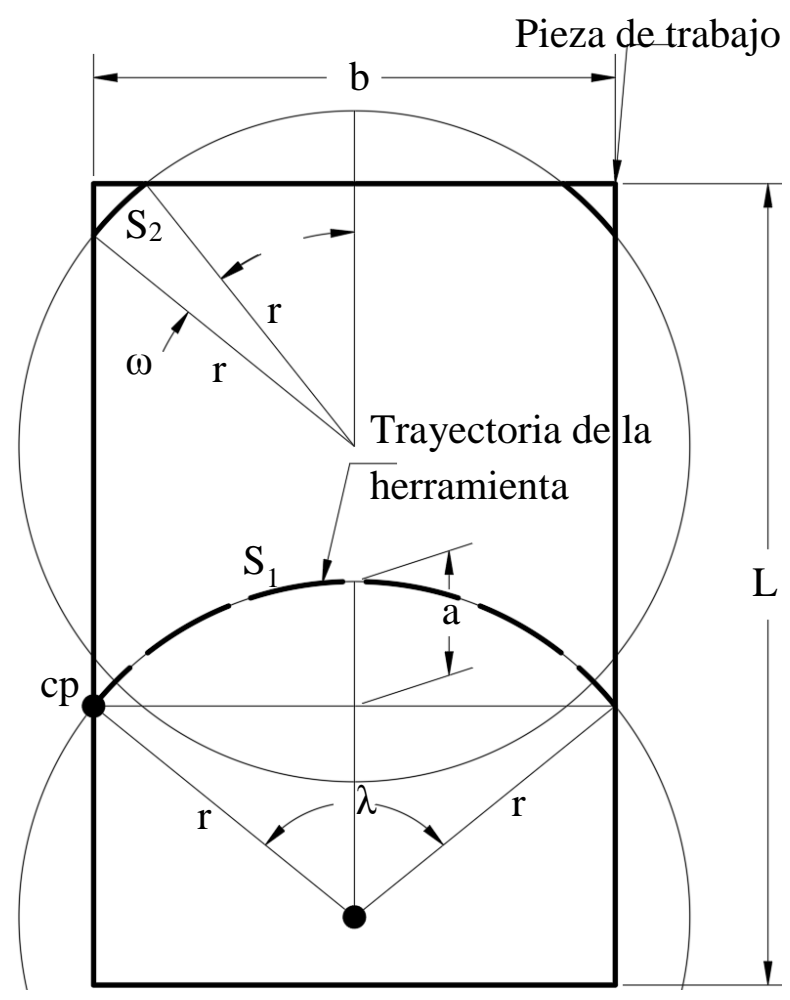

Figura 2 Análisis de la longitud de corte la Tabla 1.

Las condiciones de corte se muestran en

\begin{tabular}{|l|r|r|}
\hline \multicolumn{1}{|c|}{ Parámetro de corte } & Valor & Unidades \\
\hline Velocidad de corte $\left(V_{c}\right)$ & 900 & $\mathrm{~m} / \mathrm{min}$ \\
\hline Avance $(f)$ & 0.1 & $\mathrm{~mm} /$ diente \\
\hline Profundidad de corte $(t)$ & 1 & $\mathrm{~mm}$ \\
\hline
\end{tabular}

Tabla 1 Condiciones de corte 
El desgaste de flanco promedio se midió en cada pasada por medio de un microscopio Mitutoyo toolmaker's, al cual se le conectó una cámara Moticam de alta definición. El ángulo horizontal $\alpha$, mostrado en la Figura 3, se mantiene constante a $45^{\circ}$.

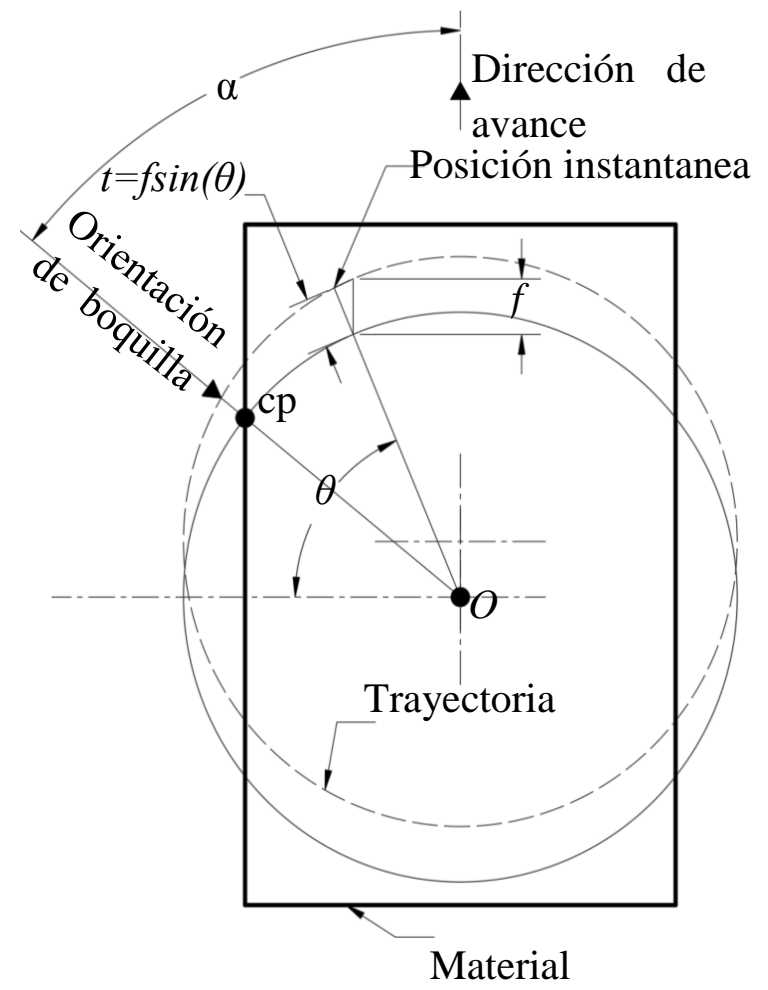

Figura 3 Representación esquemática del ángulo horizontal, $\alpha$

4.

El ángulo vertical $\varphi$ se ilustra en la Figura

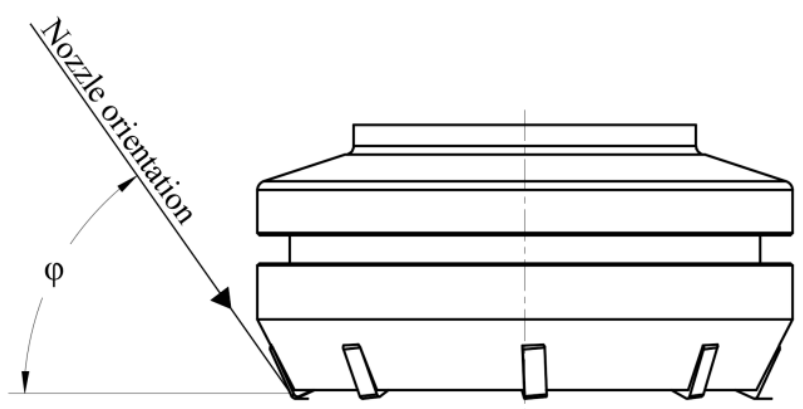

Figura 4 Representación esquemática del ángulo vertical, $\varphi$

El inserto utilizado es de geometría cuadrado con esquinas redondeadas, cuya designación ISO es SNMN120408. La herramienta de fresado frontal (comúnmente conocida como planeador) tiene una designación nombrada por el fabricante como SEK-D5.01.5-7-4HD. Los factores analizados fueron el flujo de aire, caudal de lubricante y el ángulo vertical.
El flujo de aire y la cantidad de lubricante pueden ser variados por medio del equipo MQL. Dichos factores fueron escogidos porque prácticamente son todos los factores que intervienen en el proceso. Factores no variados dentro del diseño de experimentos fueron el tipo de lubricante y el ángulo de orientación de la boquilla en el plano horizontal. Para lograr el ángulo vertical y horizontal de la forma más exacta posible, se diseñó un orientador, el cual se muestra en Figura 5.

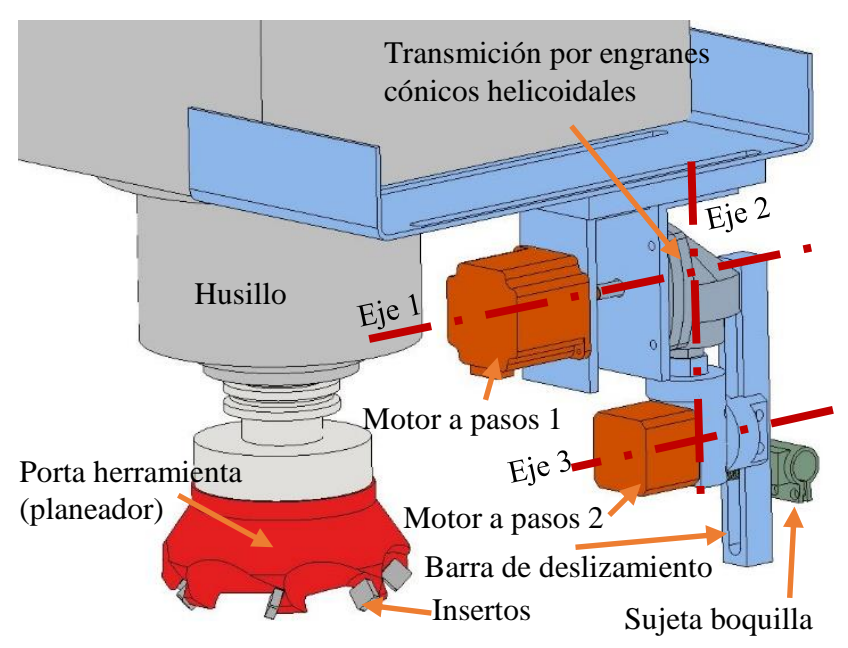

Figura 5 Modelo tridimensional del orientador.

El funcionamiento del localizador es como sigue:

1. El motor a pasos 1 está fijo por un soporte y causa rotación en un eje perpendicular a su propio eje gracias a la transmisión por engranes cónicos helicoidales, cuya relación de transmisión es de $\frac{10}{37}$.

2. El movimiento de salida de la transmisión se traduce en una rotación alrededor del eje vertical (eje 2). Esta rotación causa que el sistema inferior de rotación gire. Por lo que esta rotación está asociada al ángulo horizontal $\alpha$ (ver Figura 3).

3. El motor a pasos 2 causa que la barra de deslizamiento gire junto con el sujetador de boquilla alrededor del eje 3 .

4. Finalmente el sujetador de boquilla puede deslizarse por la barra de deslizamiento y la longitud de manguera se ajusta hasta obtener la distancia de la boquilla deseada. Para ubicar el punto deseado, es necesario recorrer el sujetador en forma manual y mantener el sujetador de boquilla a $90^{\circ}$ de la barra de deslizamiento. 


\section{Resultados y Discusión}

En la Tabla 2 se muestran los factores con los valores numéricos de sus respectivos niveles. El flujo de aire en $\mathrm{ft}^{3} /$ min y la cantidad de lubricante en $\mathrm{ml} / \mathrm{min}$, fueron evaluadas en base a gráficas proporcionadas por el fabricante del equipo. La cantidad de flujo suele darse $\mathrm{ml} / \mathrm{hr}$ debido a la poca cantidad que se aplica. Valores típicos van desde 5 a $200 \mathrm{ml} / \mathrm{h}$ (Stephenson \& Agapiou, 2016).

\begin{tabular}{|ll|c|c|}
\hline \multicolumn{2}{|c}{ Variable } & Nivel alto & Nivel bajo \\
\hline 1. & $\begin{array}{l}\text { Posición en el plano } \\
\text { vertical (A). }\end{array}$ & 40 & 15 \\
\hline 2. & Flujo de aire (B). & 7.14 SCFM & 0.7565 \\
& & SCFM \\
\hline 3. & $\begin{array}{l}\text { Caudal de lubricante } \\
\text { (C). }\end{array}$ & $0.325 \mathrm{ml} / \mathrm{min}$ & $\begin{array}{c}0.75 \\
\mathrm{ml} / \mathrm{min}\end{array}$ \\
\hline
\end{tabular}

Tabla 5 Factores y niveles utilizados en el diseño de experimentos

En la Tabla 3 se muestran los resultados del diseño de experimentos. En la Tabla 3 se sigue la convención usual en el diseño de experimentos; es decir, las letras representan los niveles alto y su ausencia representa el nivel bajo. De la Tabla 3 se puede observar que el valor más bajo de desgaste corresponde a la prueba número 3 , la cual corresponde a un nivel bajo de flujo de aire y ángulo vertical, con un nivel alto de velocidad de lubricante. Se reportan dos réplicas por cada experimento, con esto es posible una estimación interna del error y se evita el uso del llamado principio de la esparcidad de los efectos principales. Considerando las réplicas, se obtienen un total de 16 corridas.

\begin{tabular}{|l|l|r|r|}
\hline $\begin{array}{c}\text { Número de } \\
\text { prueba }\end{array}$ & \multicolumn{1}{c}{$\begin{array}{c}\text { Factores y } \\
\text { niveles }\end{array}$} & \multicolumn{2}{c|}{$\begin{array}{c}\text { Desgaste de flanco } \\
\text { promedio (mm) }\end{array}$} \\
\cline { 3 - 4 } & & Replica 1 & \multicolumn{1}{c|}{ Replica 2 } \\
\hline 1 & $a$ & 0.113 & 0.13 \\
\hline 2 & $b$ & 0.5 & 0.42 \\
\hline 3 & $c$ & 0.072 & 0.075 \\
\hline 4 & $a b$ & 0.13 & 0.135 \\
\hline 5 & $a c$ & 1.1 & 0.95 \\
\hline 6 & $b c$ & 0.143 & 0.12 \\
\hline 7 & $a b c$ & 0.8 & 0.4 \\
\hline 8 & $(-1)$ & 0.401 & 0.387 \\
\hline
\end{tabular}

Tabla 3 Resultados

Para obtener un juicio objetivo de la relevancia de cada factor, se recurrió al análisis de varianza, el cual se muestra en Tabla 4.
Del análisis de varianza, mostrado en la Tabla 4, se puede decir que la posición en el plano vertical (A), el caudal del lubricante (C) y la interacción de los factores anteriormente mencionados (AC), son factores relevantes que influyen en el desgaste.

\begin{tabular}{|l|r|r|r|r|r|}
\hline Fuente & \multicolumn{1}{|c}{$\begin{array}{c}\text { Suma de } \\
\text { cuadrados }\end{array}$} & \multicolumn{1}{c}{ GL } & \multicolumn{1}{c|}{$\begin{array}{c}\text { Cuadrado } \\
\text { medio }\end{array}$} & \multicolumn{1}{c|}{ F_q } & P_val \\
\hline $\mathrm{A}$ & 0.16810 & 1 & 0.168 & 14.159 & 0.0055 \\
\hline $\mathrm{B}$ & 0.02103 & 1 & 0.021 & 1.771 & 0.2199 \\
\hline $\mathrm{C}$ & 0.13032 & 1 & 0.130 & 10.977 & 0.0106 \\
\hline $\mathrm{AB}$ & 0.07236 & 1 & 0.072 & 6.095 & 0.0387 \\
\hline $\mathrm{AC}$ & 1.02010 & 1 & 1.020 & 85.926 & 0.00001 \\
\hline $\mathrm{BC}$ & 0.04928 & 1 & 0.049 & 4.151 & 0.07597 \\
\hline $\mathrm{ABC}$ & 0.04580 & 1 & 0.045 & 3.857 & 0.08512 \\
\hline ERROR & 0.09497 & 8 & 0.011 & 1.000 & \\
\hline TOTAL & 1.60196 & 15 & 0.106 & 8.995 & \\
\hline
\end{tabular}

Tabla 4 ANOVA

La interacción entre la posición vertical y el flujo de aire (AB) también resultó ser relevante, aunque en menor medida. Durante la parte experimental se pudo observar que al usar el nivel alto de flujo de aire, éste fluye en forma agresiva, a tal grado que resulta molesto $y$ peligroso respirar la combinación fluido de corte con aire. Además, el aire arroja la viruta con violencia, por lo que la mejor decisión es probar con un nivel bajo del factor $\mathrm{B}$.

Las variables naturales se pueden convertir a codificadas con el fin de obtener un modelo de regresión ajustado en función de los efectos y la interacción de los efectos. Las variables codificadas se calculan como sigue:

$x=\frac{\text { variable }\left(\text { variable }_{\text {bajo }}+\text { variable }_{\text {alto }}\right) / 2}{\left(\text { variable }_{\text {alto }}-\text { variable }_{\text {bajo }}\right) / 2}$

Considerando solo los efectos relevantes y las interacciones entre efectos que resultaron relevantes, es posible obtener la siguiente modelo de regresión:

$y=\beta_{0}+\beta_{1} x_{1}+\beta_{3} x_{3}+\beta_{12} x_{12}+\beta_{13} x_{13}+\varepsilon$

Donde $x_{1}$ es la variable codificada que representa la posición vertical en el plano, $x_{3}$ la variable codificada del caudal de lubricante, $x_{13}$ la interacción entre los dos efectos anteriormente mencionados y $x_{12}$ representa la interacción entre los factores posición vertical y flujo de aire. Las $\beta$ 's son los respectivos coeficientes de regresión y $\varepsilon$ es el error experimental. 
Para el análisis de los datos se construyó un programa del cual se obtiene el ANOVA y los coeficientes de la ecuación de regresión. La Tabla 6 muestra los coeficientes de todas las variables codificadas. Una ventaja de usar variables codificadas es que se visualiza de forma más simple el efecto que tiene aumentar o disminuir el valor de algún efecto en la respuesta. Adicionalmente, es posible tomar directamente solo los coeficientes asociados a los efectos y las interacciones importantes (A, C, AC y BC).

\begin{tabular}{|l|l|}
\hline \multicolumn{1}{|l|}{ Factores } & Coeficientes \\
\hline $\mathrm{A}$ & 0.1025 \\
\hline $\mathrm{B}$ & -0.03625 \\
\hline $\mathrm{C}$ & 0.09025 \\
\hline $\mathrm{AB}$ & -0.06725 \\
\hline $\mathrm{AC}$ & 0.2525 \\
\hline $\mathrm{BC}$ & -0.0555 \\
\hline $\mathrm{ABC}$ & -0.0535 \\
\hline Constante & 0.36725 \\
\hline
\end{tabular}

Tabla 6 Coeficientes de la función de respuesta en función de variables codificadas

Por otra parte, si se utilizan los coeficientes de las variables reales, las cuales se muestran en la Tabla 6, es necesario recalcular los valores de los coeficientes para el modelo de regresión reducido con variables reales en función de la ecuación del modelo reducido para variables codificadas.

\begin{tabular}{|l|l|}
\hline Factores & Coeficientes \\
\hline A & -27.09041667 \\
\hline B & -0.05984 \\
\hline C & -0.083636667 \\
\hline AB & 0.076633333 \\
\hline AC & 0.096866667 \\
\hline BC & 0.000226133 \\
\hline ABC & -0.000285333 \\
\hline Constante & 23.64575 \\
\hline
\end{tabular}

Tabla 6 Coeficientes de la función de respuesta en función de variables reales

El modelo de regresión que predice el desgaste de la herramienta es:

$$
\begin{aligned}
& V_{b}=0.36725+0.10245 * x_{1}+0.09025 * x_{3}- \\
& 0.06725 * x_{12}+0.2525 * x_{13}
\end{aligned}
$$

A partir del modelo de regresión se puede construir la superficie de respuesta, la cual se muestra en la Figura 6.

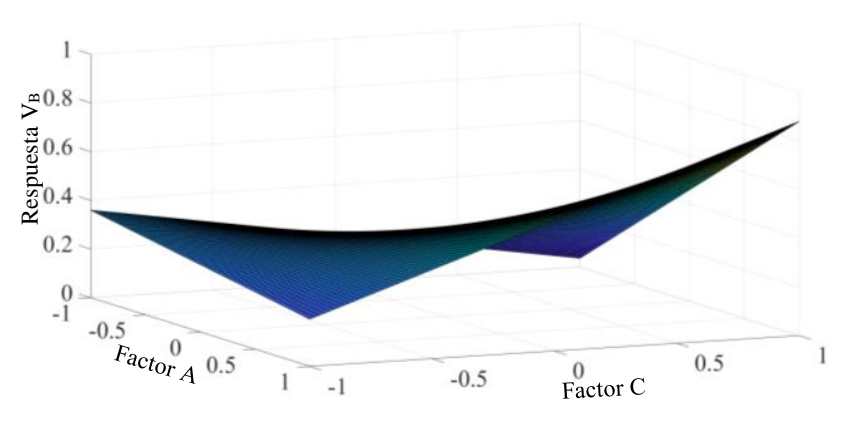

Figura 6 Superficie de respuesta para el factor B en el nivel bajo

En la Figura 6, se puede percibir cierta curvatura en la superficie de respuesta. Dicha curvatura se debe a la interacción entre factores. A partir de la superficie de la Figura 6, se graficó el contorno que se muestra en la Figura 7.

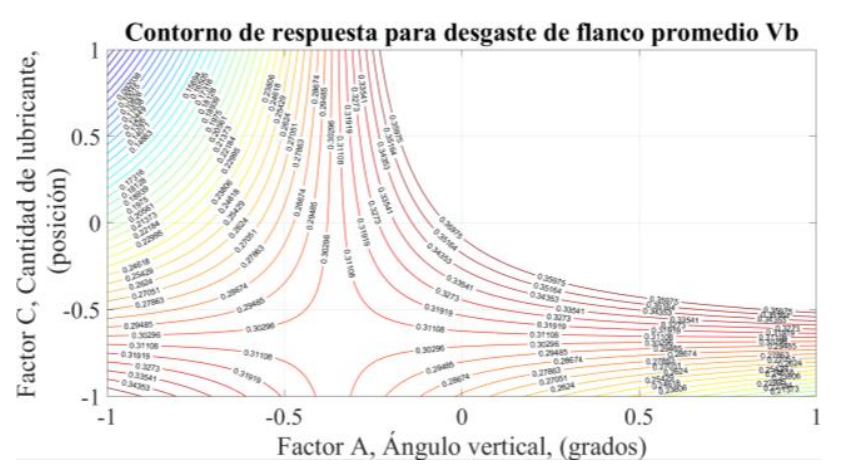

Figura 7 Contorno de respuesta para el factor B en el nivel bajo

En la Figura 7 se observa que la región con los valores más bajos de desgaste (mostrados en azul) de flanco se encuentran alrededor de la zona con el ángulo horizontal bajo y la cantidad de lubricante alta. Por el contrario al usar una cantidad de lubricante baja y un alto ángulo vertical, el desgaste de flanco resultó alto (mostrado en rojo). Dentro de los límites escogidos para los factores, es decir, los niveles escogidos para cada uno de los factores, podemos concluir que los valores óptimos están alrededor de 15 grados del ángulo vertical $\varphi$ (ver Figura 4), el flujo de aire bajo 0.8 SCFM y la cantidad de lubricante alta $0.325 \mathrm{ml} / \mathrm{min}$.

En la Figura 8 se muestran fotografías del desgaste en la herramienta cuando se usó el nivel alto en todos los factores. 


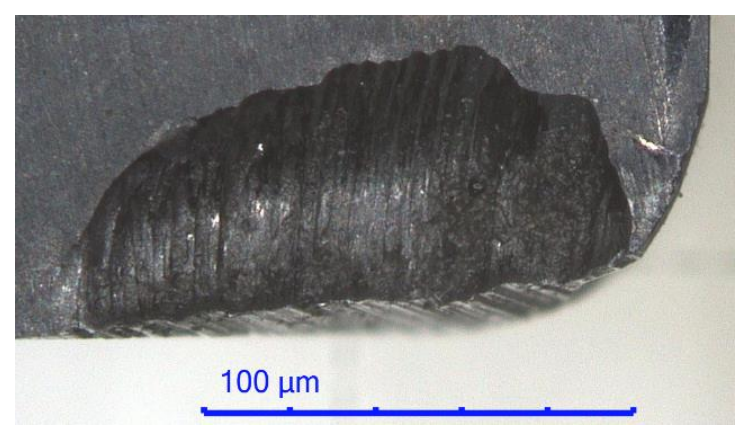

a) Desgaste de cráter (condición $\mathrm{ABC}$ ).

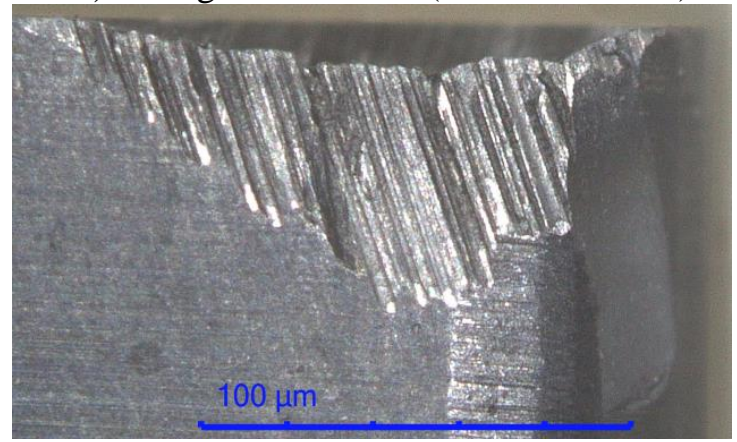

b) Desgaste de flanco (condición ABC).

Figura 8 Desgaste de cráter y flanco para la condición con todos los factores en nivel alto (ABC)

En dichas fotografías se logra observar desgaste excesivo tanto en el flanco como en la cara de arrastre. En esta situación, existe la posibilidad que la combinación de alto flujo de aire y ángulo $\varphi$ resultaran en una pobre exposición de lubricante en el borde de corte, la cara del flanco y la cara de arrastre. En general, se obtuvieron malos resultados con el ángulo $\varphi$ en un nivel alto.

En la Figura 8 se muestra el desgaste de cráter y de flanco en la herramienta cuando el caudal de lubricante está en su nivel alto y los otros dos en nivel bajo. En la Figura 8 a) se puede observar que a comparación de la Figura Figura 7a) el desgaste de cráter es considerablemente menor. Aunado al desgaste agresivo sobre el borde de corte. Respecto al desgaste de flanco, se observa que en la Figura 8 b), va desde la cara de flanco hasta el redondeo. Por otra parte, en la Figura 9 b) se puede observar que el desgaste apenas se distingue, por lo que es evidente que se logró una considerable reducción en el desgaste.

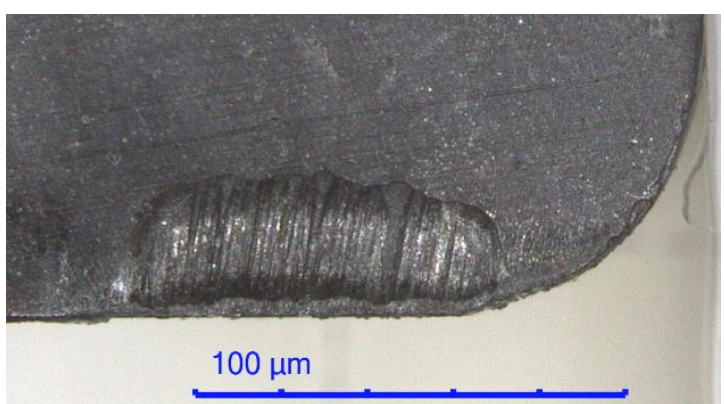

a) Desgaste de cráter (condición C).

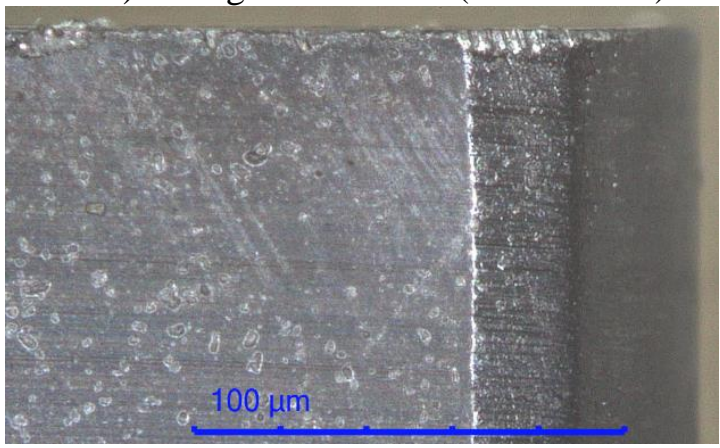

b) Desgaste de flanco (condición C).

Figura 9 Desgaste de cráter y flanco para la condición con el factor caudal de lubricante en nivel alto (condición C)

La importancia práctica de los resultados obtenidos está en el hecho de que una reducción en el desgaste implica una disminución en costos de producción relacionados con el costo de las herramientas y reducción en el costo por material de trabajo desperdiciado a causa del daño que induce una herramienta desgastada en el material de trabajo.

El aporte científico del presente proyecto está en que hasta ahora los parámetros de MQL no han sido considerados en proceso de fresado frontal utilizando técnicas estadísticas y un método sistemático que permita discernir la relevancia de cada factor y los valores que dan como resultado una disminución considerable en el desgaste de la herramienta. Más aún, la técnica de MQL es poco conocida.

Los resultados demuestran que los parámetros de MQL tienen efecto en la eficacia del sistema MQL. Sin embargo, se probaron en un proceso. A futuro se debe extender el estudio a otros procesos de maquinado.

\section{Conclusión}

Una serie de experimentos fueron realizados en el fresado frontal de AISI1045 a alta velocidad utilizando MQL y variando tres factores en dos niveles. De los resultados reportados se puede concluir lo siguiente: 
1. El diseño y la fabricación del dispositivo orientador fue de importancia para asegurar factores importantes, tal como fue el caso del ángulo vertical.

2. El análisis de varianza reveló que el ángulo vertical y la cantidad de lubricante son factores importantes, así como su interacción. Sin embargo, la interacción entre el flujo de aire y el ángulo resultó ser relevante según el ANOVA. Se recomienda utilizar el flujo de aire bajo para evitar problemas con la viruta e inhalar el lubricante suspendido en el aire.

3. El contorno de superficie de respuesta revela la zona de optimización, resultando que los valores recomendados para minimizar el desgaste de flanco promedio son: 15 grados del ángulo vertical $\varphi$ (ver Figura 6), el flujo de aire bajo 0.8 SCFM y la cantidad de lubricante alta $0.325 \mathrm{ml} / \mathrm{min}$. Con estos parámetros se logró hasta 7 veces menos desgaste de flanco de la herramienta de corte.

\section{Referencias}

Bruni, C., d`Apolito, L., Focellese, A., Gabrielli, F., \& Simoncini, M. (2008). Surface roughness modelling in finish face milling under MQL and dry cutting conditions. International Journal of material forming, 1, 503-506.

Childs, T., Maekawa, K., Obikawa, T., \& Yamane, Y. (2000). Metal machining theory and applications. London: Arnold.

Heisel, U., Lutz, M., Spath, D., Wassmer, R., \& Walter, U. (1994). Application of minimum quantity cooling lubrication technology in cutting process. Prod. Eng., II/I, 49-54.

Jang, D.-y., Jung, J., \& Seok, J. (2016). Modeling and parameter optimization for cutting energy reduction in MQL milling process. International Journal of Precision Engineering and Manufacturing-Green Technology, 3(1), 512.

Kasim, M., Che Haron, C. H., Ghani, J. A., \& Sulaiman, M. A. (2013). Prediction Surface Roughness in High-Speed Milling of Inconel 718 under Mql Using Rsm Method. Middle-East Journal of Scientific Research, 13(3), 264-272.
Le Coz, G., Marinescu, M., Devillez, A., Dudzinski , D., \& Velnom, L. (2012). Measuring temperature of rotating cutting tools: Application to MQL drilling and dry milling of aerospace alloys. Applied thermal engineering, $36,434-441$.

Liu, Z. Q., Cai, X. J., Chen, M., \& An, Q. L. (2011). Investigation of cutting force and temperature of end-milling $\mathrm{Ti}-6 \mathrm{Al}-4 \mathrm{~V}$ with different minimum quantity lubrication (MQL) parameters. Proceedings of the Institution of Mechanical Engineers, Part B: Journal of Engineering Manufacture, 225(8), 1273-1279.

Mia, M., Al Bashir, M., Kan, M., \& Dhar, N. R. (2017). Optimization of MQL flow rate for minimum cutting force and surface roughness in end milling of hardened steel (HRC 40). The international Journal of Advanced Manufacturing Technology, 89(1-4), 675-690.

Sharma, A. K., Tiwari, A. K., \& Dixit, R. A. (2016). Effects of Minimum Quantity Lubrication (MQL) in machining processes using conventional and nanofluid based cutting fluids: A comprehensive review. Journal of Cleaner Production, 127(20), 1-18.

Singh, G., Gupta, M. K., Mia, M., \& Sharma, V. S. (2018). Modeling and optimization of tool wear in MQL-assisted milling of Inconel 718 superalloy using evolutionary techniques. The International Journal of Advanced Manufacturing Technology, 97(1-4), 481-494.

Stephenson, D. A., \& Agapiou, J. S. (2016). Metal cutting theory and practice. Boca Ratón: CRC Press Taylor \& Francis Group.

Tawaloki, T., Hadad, M. J., \& Sadeghi, M. H. (2010). Influence of oil mist parameters on minimum quantity lubrication - MQL grinding process. International Journal of Machine Tools and Manufacture, 50(6), 521-531.

Wakabayashi, T., Sato, H., \& Inasaki, I. (1998). Turning using extremely small amount of cutting fluids. JSME, 41, 143-148.

Wang, C. D., Cheng, M., An, Q.-L., Wang, M., \& Zhu, Y.-H. (2014). Tool wear performance in face milling Inconel 182 using minimum quantity lubrication with different nozzle positions. International Journal of Precision Engineering and Manufacturing, 15(3), 557565 .

MARTÍNEZ-RAMÍREZ, Israel, GUTIÉRREZ-RIVERA, Miguel Ernesto, ESPINOZA-TORRES, Isaí y RODRÍGUEZ-FLORES, Javier. Efecto de parámetros de mínima cantidad de lubricación en reducción de desgaste de herramienta. Revista de Ingeniería Mecánica. 2019. 